\title{
An Improved Hybrid Framework for Evaluating a Mobile Device Location in a Wireless Network
}

\author{
Adekunle A. Adeyelu \\ Department of Mathematics \& \\ Computer Science \\ Benue State University, Makurdi
}

\author{
Onaji J. Onah \\ Department of Mathematics \& \\ Computer Science \\ Benue State University, Makurdi
}

\author{
Iwuese J. Orban \\ Department of Mathematics \& \\ Computer Science \\ Benue State University, Makurdi
}

\begin{abstract}
Studies on techniques to track an object from a remote location have been ongoing for several years. Existing outdoor solutions to locate a Mobile Station (MS) within a cellular network require optimization in terms of accuracy and latency. In this study, an Enhanced Mobile Station Positioning (EMSP) architecture for a Wireless Sensor Network was developed and its performance was appraised using accuracy and latency metrics. The model is a fusion of the Received Signal Strength (RSS) and Time Difference of Arrival (TDOA) techniques. The RSS used the strength of the signal received at four Base Stations (BS) positioned within the neighborhood of the MS to locate it while the TDOA paradigm utilized the difference in arrival time of the signals to locate the MS. The RSS forms a circle on which the MS can be traced while the TDOA offers a hyperbola on which the MS can be located. The mathematical model was derived by solving both the circles and the hyperbolas with Taylor's series expansion formula. The estimated position of the MS was determined using Linear Least Square (LLS) solution in a repetitive manner. The performance of the simulated model was evaluated using accuracy and latency metrics. The result showed that a MS was located within error distances of $115 \mathrm{~m}$ for $67 \%$ and $152 \mathrm{~m}$ for $95 \%$ of the time it was deployed. This result outclassed the same technique using three BS (as benchmark) which located the MS within $128 \mathrm{~m}$ at $67 \%$ deployment and $175 \mathrm{~m}$ at $95 \%$ of the time, an $11.6 \%$ improvement in accuracy. The results also revealed that the latency experienced when the BSs were increased from three to four increased by $22.67 \%$. It can be concluded that increasing the number of BSs from three to four gave a significant improvement in accuracy in locating a MS within the BSs.
\end{abstract}

\section{General Terms}

Mobile Station Positioning techniques, GSM localization

\section{Keywords}

Mobile Station, Base Station, Received Signal Strength, Time Difference of Arrival, Hybrid techniques

\section{INTRODUCTION}

With the aid of wireless networks, digital communication has evolved pretty well within the last few decades. No doubt it has been a radical pattern shift that enabled multimedia communications between people and devices from any location. Mobile wireless communication system is an example of Cellular communication. Cellular communication is accomplished using Global System for Mobile communications (GSM) devices. GSM currently is confronted with many issues. These challenges includes high energy consumption, security issues among others. Another major challenge of GSM is finding the accurate position of a MS or
GSM device within a GSM network. Any technique deployed to find the location of an MS within a network with respect to some known location information is called Mobile Station Positioning (MSP) technique.

For several hundred years now, methods of finding the position of a remote object with reference to a known location have been needed. In [1] it was reported that these methods were first functional in road surveying, target ranging for weaponry, and in map making. Since there has been a huge increase in the number of MS users within the last 10 years, the number of applications that require location information is growing swiftly and MSP service in cellular networks has become a hot topic among researchers [2].

Applications that render services based on the knowledge of the MS position are called Location Based Service (LBS). MSP techniques are therefore the backbone upon which LBS thrives. The main drive behind the development of MSP techniques to support (LBS) in cellular networks was the need to trace the origin of emergency calls (E-911) made from MS [3]. At present, this initiative is now being applied in different areas including location sensitive billing, location based marketing, location of nodes in a distributed sensor network, intelligent traffic system, weather applications, military artillery and lots more.

Many existing single techniques have been proposed to estimate location of MS. Examples include Global Positioning System (GPS), Angle of Arrival (AOA), Received Signal Strength (RSS), Time of Arrival (TOA), Time Difference of Arrival (TDOA), Signal Attenuation Difference of Arrival (SADOA), Cell Identification methods (Cell-ID). Other (hybrid) techniques include RSS/TDOA, TOA/AOA, AOA/TDOA, SADOA/TDOA etc. They combine the strengths of individual techniques to make up a hybrid which outperforms each constituent technique. RSS is a simple to calculate and easy to implement technique, however it is not the preferred choice in terms of accuracy. On the other hand, TDOA is the leading technique among the individual estimation schemes but meets its frustration in the presence of obstacles in Non Line of Sight environment.

The Federal Communications Commission (FCC) of the United States of America issued out its accuracy standard in 2001 compelling all MSP service providers to work towards achieving this standard. The FCC accuracy requirement states that $67 \%$ of readings produced by any given (outdoor) MSP technique should be within 100 meters of the MS and $95 \%$ of the readings within 300 meters of the MS [4], [2]. The aim of every MSP technique therefore is to meet the FCC accuracy requirement without necessarily consuming too much infrastructure or network resources in the process. The challenge lies in the fact that the accuracy of the existing 
(Network-based) outdoor positioning techniques is insufficient as they have not met the accuracy requirement of the FCC especially under the Non Line of Sight (NLOS) environments. Efforts are still being made in this regard. In [2], RSS/TDOA with 3 BS was studied, they however did not study the effect of an additional BS and the latency issues that accompany it. In [5], RSS technique was enhanced with 4 BS, while [6] enhanced the TDOA using 4 BS. This has motivated the authors to combine the RSS/TDOA with measurements from 4 BS (as against the conventional 3 BS). This study therefore attempts to enhance accuracy while investigating the latency.

The rest of the paper is as follows. Section 2 discusses some key related works by other authors, in section 3 , the TDOA mathematical model is derived, the methodology for the model is described in detail, and the Algorithm is shown. In section 4, the accuracy and latency results are presented and discussed. Based on these results, conclusions are made in 5 . The paper closes by suggesting future works in section 6 .

\section{LITERATURE REVIEW}

Due to the dramatic boom in MS usage within the last decade, MSP in cellular networks has become a hot topic among researchers. Some notable contributions by some of these authors are reviewed in this section.

Object location tracking started with the integration of the GPS technology with mobile internet. This made outdoor localization accomplish great success in map navigation, people location, object tracking, etc. However, GPS technology meets its frustration in indoor applications because of the great attenuation of the satellite signal and multipath effect caused by the obstruction from buildings and the complex indoor environment [5].

There are two different approaches in position estimation techniques as presented in [7]: single estimation techniques and hybrid schemes. The single techniques include AOA, TDOA, TOA and RSS. The hybrid schemes for position estimation presented in the literature includes TOA/RSS, TDOA/RSS, TDOA/AOA and AOA/TOA. The literature showed that hybrid schemes tend to outperform single schemes. However, the more accurate a constituent technique of a hybrid is, the more accurate the hybrid will be. There was an improvement in accuracy on the RSS [5] and on TDOA using 4 BS. TDOA technique was also studied in [8] and [9]. Specifically, the authors studied localization accuracy using Time Difference of Arrival (TDOA) measurements within a sensor network. Their results confirm high accuracy and show the better performance of an additional BS. The hybrid pair RSS/TDOA was extensively studied in [2] but they did not investigate the latency. Their technique used 3 BSs. According to their model the strength of the signal received at the BS, is used to find the distance between the MS and BSs. The time difference of arrival of signal exchanged between the MS and the BSs forms a hyperbola on which the MS lies. Solving the intersection of the circles and hyperbolas gives the location of the MS. [2], [7] reported that increasing the BS would be beneficial in terms of accuracy. This paper therefore studies the accuracy of the RSS/TDOA taking measurements from 4 BS (instead of the conventional 3 BS) and further investigates the latency issues that arises due to the additional BS.

\section{PROPOSED HYBRID TECHNIQUE}

\subsection{Conceptual Model}

This section explains the conceptual model of the proposed technique. Figure 1 shows the conceptual model. First the RSS technique produces a circle centered at the BS. The MS can be located anywhere on the circumference of the circle. However, using one BS does not specify which exact direction from the center the MS can be located. This technique therefore uses four $\mathrm{BSs}$ (denoted $\mathrm{BS}_{1}, \mathrm{BS}_{2}, \mathrm{BS}_{3}$, and $\mathrm{BS}_{4}$ ) and produces four circles centered at each BS. The point of intersection between all four BS provides the initial location estimate of the MS $\left(x_{0}, y_{0}\right)$.

Anywhere between any two fixed points $\mathrm{BS}_{1}$ and $\mathrm{BS}_{2}$ that the MS roams, it follows that the difference in the distance between the $\mathrm{MS}$ and $\mathrm{BS}_{1}$ and $\mathrm{MS}$ and $\mathrm{BS}_{2}$ is constant. Therefore this set of points between the MS and the fixed BSs forms a hyperbola with $\mathrm{BS}_{1}$ and $\mathrm{BS}_{2}$ as the foci. The MS resides on the hyperbola. Given $\mathrm{BS}_{1}$ as a reference point for example, taking readings from $\mathrm{BS}_{1}, \mathrm{BS}_{2}, \mathrm{BS}_{3}$ and $\mathrm{BS}_{4}$ produces three hyperbolas. Since one hyperbola is not sufficient to produce the exact point on which the MS resides, we have combined the four BSs and the MS location is at the point of intersection of the $3(\mathrm{~N}-1$, where $\mathrm{N}=4 \mathrm{BSs})$ hyperbolas.

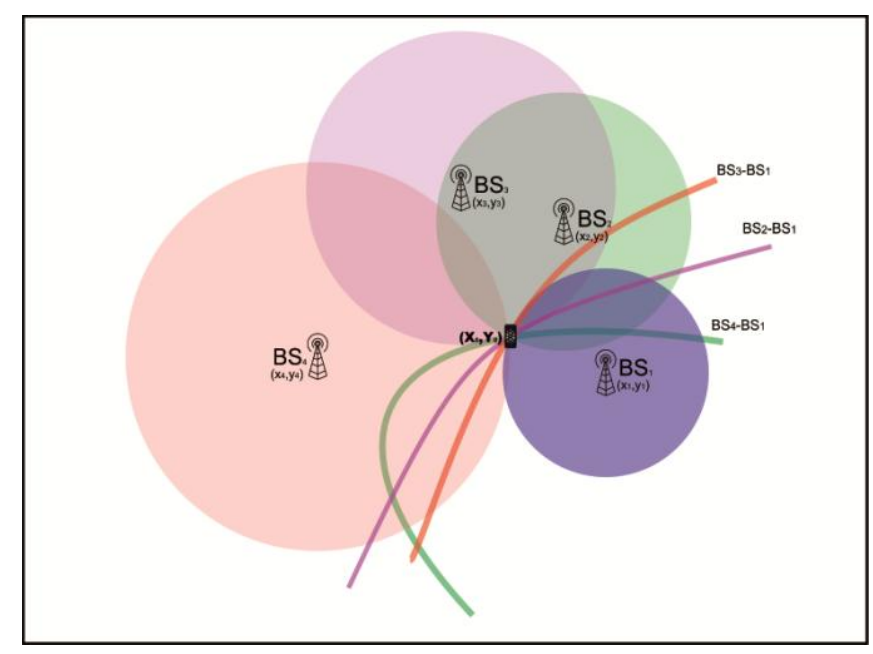

Fig 1. Conceptual Model

LLS solution is used to solve the hyperbola by using the first 2 terms of their Taylors series expansion. The LLS solution is done iteratively using $\left(x_{0}, y_{0}\right)$ obtained from the RSS as the initial estimate. The current $\left(x_{0}, y_{0}\right)$ is fed back into the LLS solution until we obtain an estimate close enough to the exact location.

The idea here is that both the knowledge of the RSS and TDOA solutions are combined to arrive at the proposed hybrid technique. These is done using the first two terms of their Taylors series expansion.

\subsection{Mathematical Model}

Expressing the set of linear equations obtained from the RSS solution as presented in [5] and the TDOA in [6] in matrix form, we have a merged (hybrid solution). 
$\mathrm{A} \hat{X}_{\mathrm{MS}}=\mathrm{B}$

where

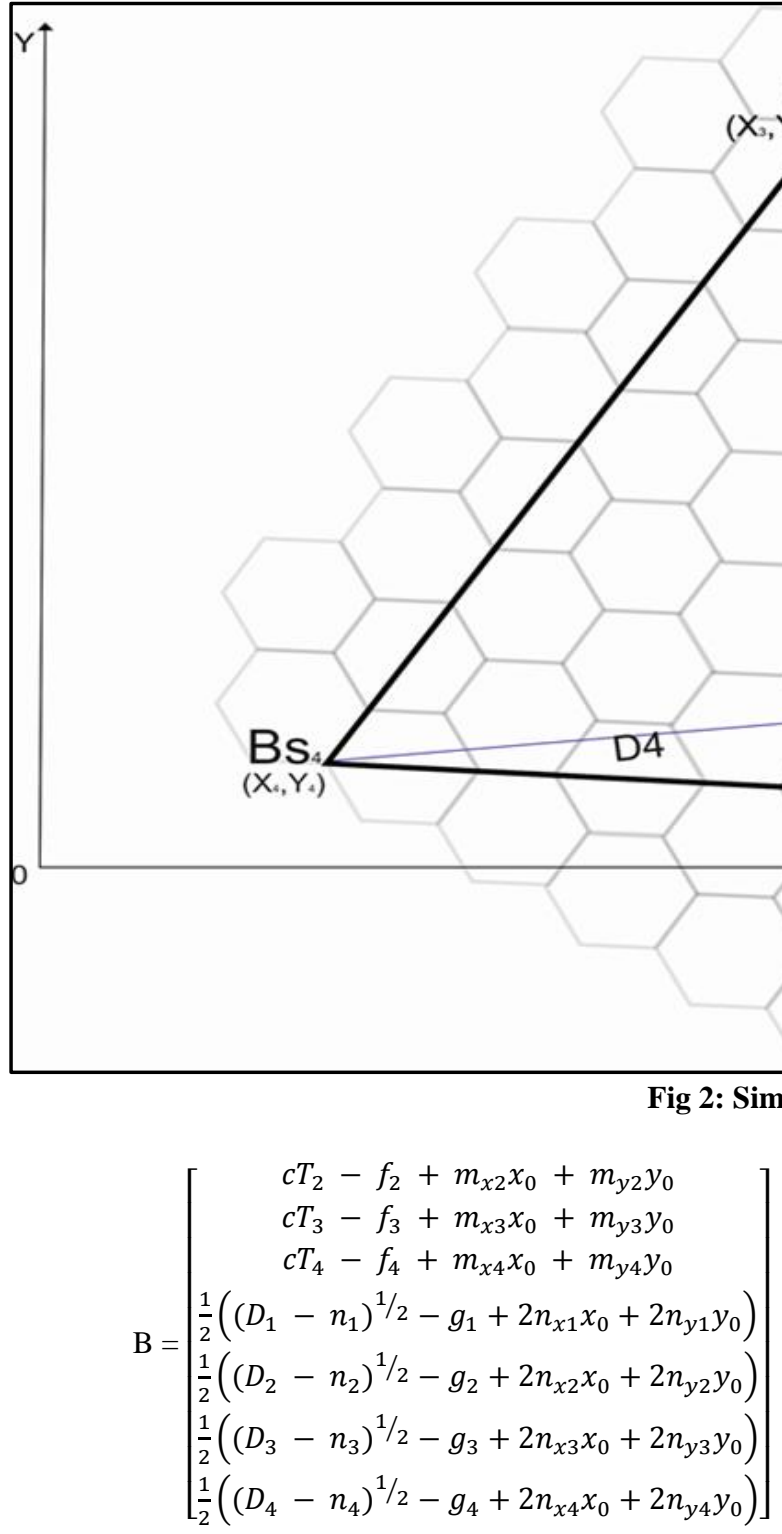

where:

$m_{y i}=\frac{\left(y_{0}-y_{i}\right)}{\sqrt{\left(x_{0}-x_{i}\right)^{2}+\left(y_{0}-y_{i}\right)^{2}}}-\frac{\left(y_{0}-y_{1}\right)}{\sqrt{\left(x_{0}-x_{1}\right)^{2}+\left(y_{0}-y_{1}\right)^{2}}}$

$n_{x i}=\left(x_{0}-x_{i}\right), n_{y i}=\left(y_{0}-y_{i}\right), g_{i}=\left(x_{0}-x_{i}\right)^{2}+$

$\left(y_{0}-y_{i}\right)^{2}$

$m_{x i}=\frac{\left(x-x_{i}\right)}{\sqrt{\left(x_{0}-x_{i}\right)^{2}+\left(y_{0}-y_{i}\right)^{2}}}-\frac{\left(x_{0}-x_{1}\right)}{\sqrt{\left(x_{0}-x_{1}\right)^{2}+\left(y_{0}-y_{1}\right)^{2}}}$

$f_{i}=\sqrt{\left(x_{0}-x_{i}\right)^{2}+\left(y_{0}-y_{i}\right)^{2}}-\sqrt{\left(x_{0}-x_{1}\right)^{2}+\left(y_{0}-y_{1}\right)^{2}}$

$x_{i}, y_{i}$ are the coordinates of the ith BS. $D_{i}$ is the distance between the MS and the ith BS, $x_{0}, y_{0}$ are the coordinates for initial position of the MS.
$\mathrm{A}=\left[\begin{array}{cc}m_{x 2} & m_{y 2} \\ m_{x 3} & m_{y 3} \\ m_{x 4} & m_{y 4} \\ n_{x 1} & n_{y 1} \\ n_{x 2} & n_{y 2} \\ n_{x 3} & n_{y 3} \\ n_{x 4} & n_{y 4}\end{array}\right], \quad \hat{X}_{\mathrm{MS}}=\left[\begin{array}{l}x \\ y\end{array}\right]$, 
intersection of this circles following the mathematical model explained in [1] produces an estimate for RSS which is further refined using TDOA formula. The TDOA produces three hyperbolas and their point of intersection produces the initial estimate for the MS position.

The LLS is further used to solve the matrices resulting from the linearization of both techniques. The location performance was assessed in terms of the value of the distance error (Root Mean Square Error) defined by:

$E_{d}=\sqrt{\left(x_{M S}-\hat{x}_{M S}\right)^{2}+\left(y_{M S}-\hat{y}_{M S}\right)^{2}}$

where $\left(x_{M S}, y_{M S}\right)$ and $\left(\hat{x}_{M S}, \hat{y}_{M S}\right)$ are the actual and estimated MS locations, respectively [3]. After several free runs of proposed model for random noise values between MS and BS, results are as presented in section 4.

\subsection{Algorithm of the System}

The algorithm of the proposed model is given in this section. Some assumptions were made as stated.

\subsubsection{Overall Algorithm of the System}

PRE: $\mathrm{x}_{\mathrm{n}}, \mathrm{y}_{\mathrm{n}}$ are the coordinates of the $\mathrm{n}^{\text {th }}$ base station. The phone to be located is assumed to be a Mobile Phone object (MS) with the following properties. MS identity (timsi), Serving base station of the MS (sbs), a list of neighboring base stations $\left(\mathrm{nbs}_{\mathrm{i}, \mathrm{j}}\right)$ where $1<=\mathrm{i}<=6$ and $1<=\mathrm{j}<=2$, a list of neighboring sorted base stations $\left(\mathrm{nsbs}_{\mathrm{i}, \mathrm{j}}\right)$ where $1<=\mathrm{i}<=4$ and $1<=\mathrm{j}<=2$, The base stations have the following nested properties: signal strength, Base Station Code (cid), latitude $(\mathrm{x})$, longitude $(\mathrm{y})$

POST: This algorithm calculates the location of a Mobile Station $X_{\mathrm{ms}}$ using the parameters in PRE and the formula in equation 2.

Step 1: Start

Step 2: Identify the calling Mobile Station Object (MS)

Step 3: Get MS.sbs

Step 4: Set $i=1$

Step 5: Repeat step 6 to 8 while i $<=6$

Step 6: Set nbs[i,1] =

getSignalStrengthDBM(MS.getNextnbs());

Step 7: Set nbs[i,2] = getCID(MS.getNextnbs ()$)$;

Step 8: Set $i=i+1$

Step 9: Sort nbs with the bubble sort algorithm in descending order (see 3.4.2.)

Step 10: $\mathrm{nsbs}[1,1]=$ getSignalStrengthDBM(MS.sbs);

Step 11: nsbs[1,2] = getCID(MS.sbs);

Step 12: Set i to 2

Step 13: Repeat step 14 to 16 while $\mathrm{i}<4$

Step 14: Set nsbs[i,1] = get nbs[i-1,1]

Step 15: Set nsbs[i,2] = get nbs[i-1,2]
Step 16: Set $\mathrm{i}=\mathrm{i}+1$

Step 17: Feed the content of nsbs into equation 2 to produce $\mathrm{X}_{\mathrm{ms}}$

Step 18: Exit

\subsubsection{Bubble Sort Algorithm (Descending Order)}

PRE: Assume list is an unsorted array of $\mathrm{n}$ elements.

POST: This algorithm returns the sorted version of list in descending order.

Step 1: Start

Step 2: for all elements of list Repeat step 3 to 6

Step 3: if list $[\mathrm{i}]<\operatorname{list}[\mathrm{i}+1]$ Repeat step 4 to 6

Step 4: temp = list[i];

Step 5: list $[\mathrm{i}]=\operatorname{list}[\mathrm{i}+1]$

Step 6: $\operatorname{list}[i+1]=$ temp;

Step 7: Exit

\subsection{Assumptions taken in model Formulation}

The following are the assumptions made in formulating the model used in this work.

1. Multi-directional BSs were used. This means the BSs send and receive signal to and from the MS in every direction.

2. The closer a BS is to the MS the stronger the signal strength as measured by the Received Signal Strength Indicator (the device used to measure the strength of a signal, usually in decibel-meter).

3. Four BSs with the highest signal strengths were used in the model for the estimation of the MS location.

4. The BSs were arranged in an orthogonal fashion with the MS in between. The distance between the MS and each BS varies and so does the corresponding signal strength.

\section{RESULTS AND DISCUSSION}

\subsection{Simulation Data Set}

The various $E_{d}$ calculated for each iteration and conveyed in Cumulative Distribution Function (CDF) measured in percentage. The corresponding Location Distance for each CDF value was also calculated. The result of the simulation is presented in Table 1 and Table 2 below for 3 BS and 4 BS respectively.

Table 1. RSS/TDOA 3 BS data set

\begin{tabular}{|c|c|}
\hline Location Distance(m) & CDF (\%) \\
\hline 0.037 & 0.008752 \\
\hline 23.100 & 2.069536 \\
\hline 24.500 & 3.157772 \\
\hline 38.500 & 12.26561 \\
\hline 46.200 & 16.38718 \\
\hline 50.000 & 21.66169 \\
\hline 51.200 & 31.66429 \\
\hline 52.800 & 34.33929 \\
\hline
\end{tabular}




\begin{tabular}{|c|c|}
\hline 53.100 & 35.64464 \\
\hline 54.700 & 37.68929 \\
\hline 58.100 & 41.76964 \\
\hline 62.200 & 42.91429 \\
\hline 66.500 & 44.13036 \\
\hline 70.500 & 45.36786 \\
\hline 78.100 & 46.64464 \\
\hline 85.100 & 47.99107 \\
\hline 90.300 & 49.74107 \\
\hline 103.500 & 50.49464 \\
\hline 108.400 & 53.25714 \\
\hline 115.000 & 54.19643 \\
\hline 119.200 & 57.43750 \\
\hline 123.400 & 59.72857 \\
\hline 124.600 & 62.15893 \\
\hline 128.000 & 67.67143 \\
\hline 131.300 & 69.21607 \\
\hline 139.500 & 71.95179 \\
\hline 141.200 & 77.70893 \\
\hline 145.800 & 80.50714 \\
\hline 154.700 & 89.54286 \\
\hline 159.200 & 91.00357 \\
\hline 163.800 & 92.53393 \\
\hline 165.700 & 93.10893 \\
\hline 175.500 & 95.68571 \\
\hline 176.200 & 97.26429 \\
\hline 179.200 & 101.9589 \\
\hline 186.000 & 103.0120 \\
\hline 193.000 & 105.3125 \\
\hline 198.000 & 107.6130 \\
\hline 210.000 & 109.9136 \\
\hline 221.000 & 112.2141 \\
\hline 228.000 & 114.5146 \\
\hline 230.000 & 116.8152 \\
\hline 237.000 & 119.1157 \\
\hline 248.000 & 121.4162 \\
\hline 256.000 & 123.7168 \\
\hline 265.000 & 126.0173 \\
\hline 276.000 & 128.3179 \\
\hline 289.000 & 130.6184 \\
\hline 300.000 & 132.9189 \\
\hline 318.000 & 135.2195 \\
\hline 331.000 & 137.5200 \\
\hline 420.000 & 139.8205 \\
\hline
\end{tabular}

Table 2. RSS/TDOA 4 BS data set

\begin{tabular}{|c|c|}
\hline Location Distance(m) & CDF (\%) \\
\hline 12.446 & 0.008752 \\
\hline 23.100 & 2.069536 \\
\hline 24.500 & 3.157772 \\
\hline 25.500 & 9.220800 \\
\hline 30.200 & 10.50330 \\
\hline 34.000 & 12.26561 \\
\hline 40.200 & 18.38718 \\
\hline 46.800 & 22.66169 \\
\hline 50.000 & 38.64464 \\
\hline 56.000 & 42.68929 \\
\hline 57.000 & 43.76964 \\
\hline 59.200 & 44.91429 \\
\hline
\end{tabular}

\begin{tabular}{|c|c|}
\hline 60.500 & 45.13036 \\
\hline 65.500 & 45.36786 \\
\hline 72.100 & 46.64464 \\
\hline 83.100 & 49.99107 \\
\hline 95.300 & 51.74107 \\
\hline 98.000 & 52.49464 \\
\hline 102.400 & 53.25714 \\
\hline 106.000 & 54.19643 \\
\hline 111.200 & 57.43750 \\
\hline 113.400 & 59.72857 \\
\hline 114.600 & 62.15893 \\
\hline 115.600 & 67.67143 \\
\hline 121.300 & 69.21607 \\
\hline 126.500 & 71.95179 \\
\hline 134.200 & 77.70893 \\
\hline 136.800 & 80.50714 \\
\hline 146.700 & 89.54286 \\
\hline 147.200 & 91.00357 \\
\hline 149.800 & 92.53393 \\
\hline 151.700 & 93.10893 \\
\hline 152.500 & 95.68571 \\
\hline 154.200 & 97.26429 \\
\hline 169.200 & 101.9589 \\
\hline 172.500 & 104.0366 \\
\hline 183.600 & 106.8495 \\
\hline 192.750 & 109.6623 \\
\hline 201.900 & 112.4752 \\
\hline 211.050 & 115.2880 \\
\hline 220.200 & 118.1009 \\
\hline 229.350 & 120.9137 \\
\hline 238.500 & 123.7266 \\
\hline 247.650 & 126.5395 \\
\hline 256.800 & 129.3523 \\
\hline 265.950 & 132.1652 \\
\hline 275.100 & 134.9780 \\
\hline 284.250 & 137.7909 \\
\hline 303.400 & 140.6037 \\
\hline 322.550 & 143.4166 \\
\hline 331.700 & 146.2295 \\
\hline 500.000 & 149.0423 \\
\hline
\end{tabular}

\subsubsection{Accuracy Result}

In this section, the dataset in Table 1 and Table 2 were plotted for stress-free analysis. The performance of each technique is analyzed based on the CDF of distance error (location distance) mainly at $67 \%$ and $95 \%$. This result reveals by how much this proposed solution approached the FCC accuracy requirement. 


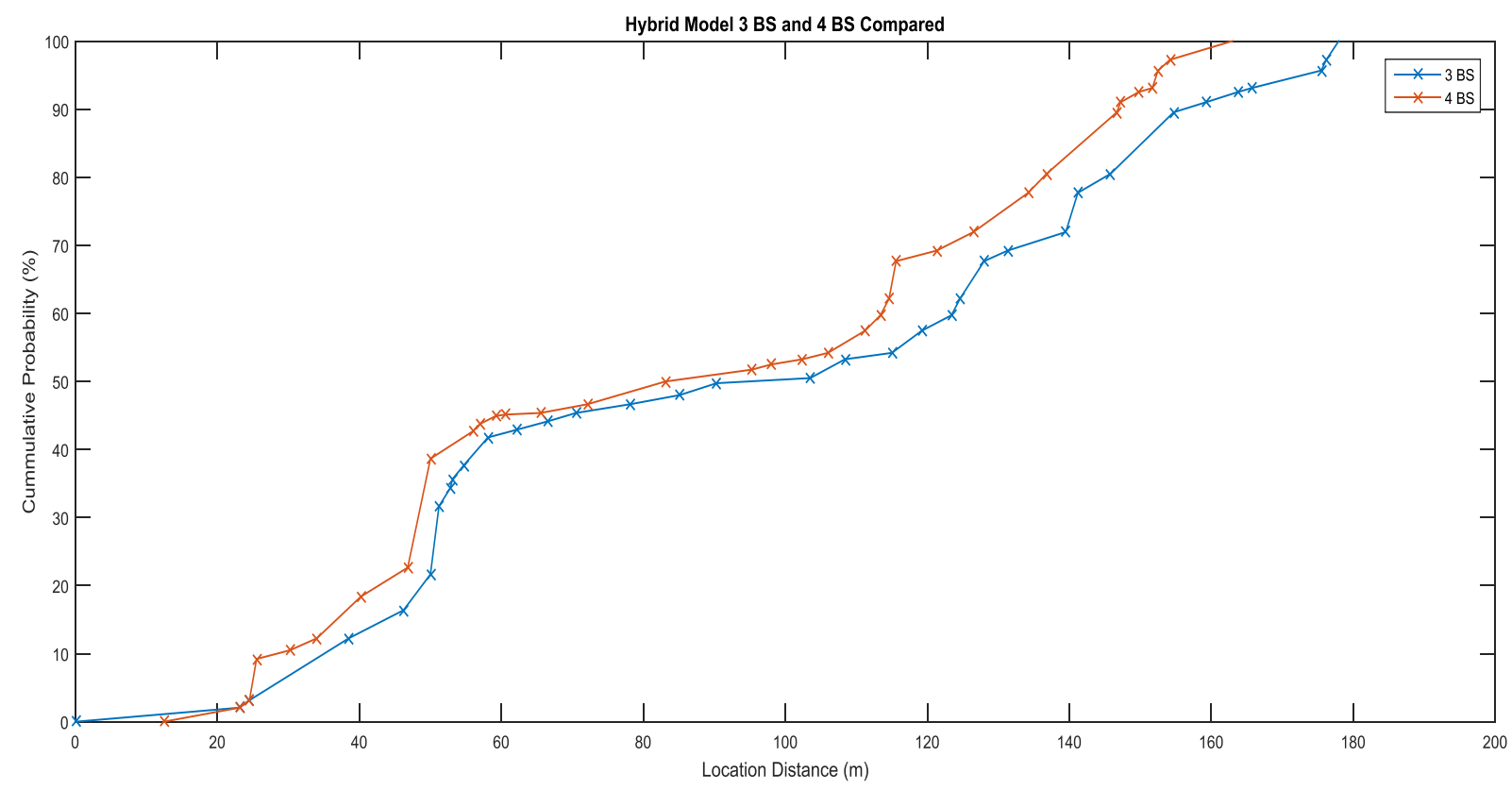

Fig 3: Hybrid Model 3 BS and 4 BS graph

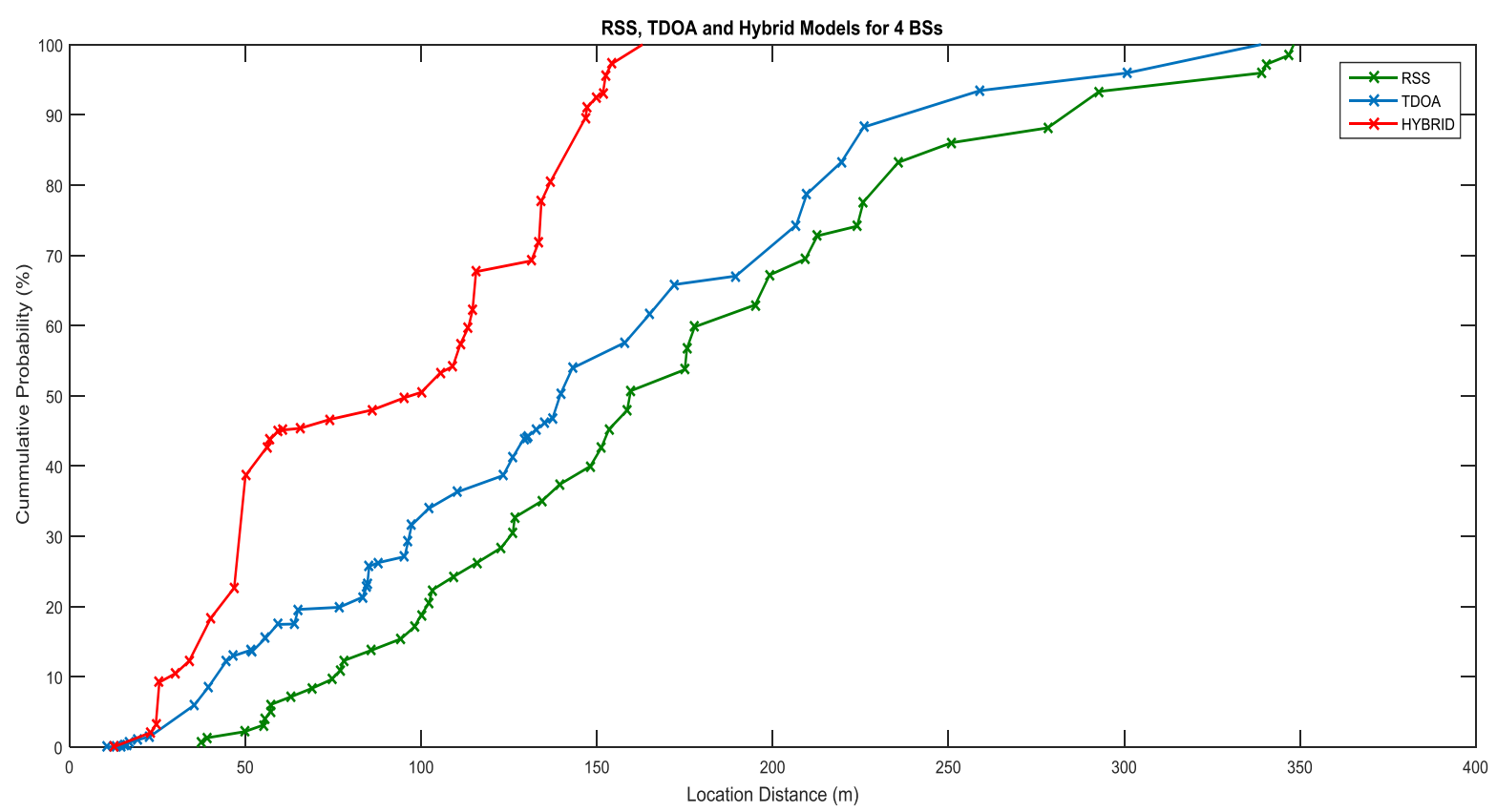

Fig 4: Graph of RSS, TDOA and Hybrid Models for 4 BS

Table 3. Accuracy Result 1: Hybrid model 3BS and 4BS

\begin{tabular}{|c|c|c|c|}
\hline $\begin{array}{c}\text { CDF } \\
(\boldsymbol{\%})\end{array}$ & $\begin{array}{c}\text { RSS/TDOA } \\
\text { HYBRID }\end{array}$ & $\begin{array}{c}\text { Location } \\
\text { Distance(m) }\end{array}$ & $\begin{array}{c}\text { Enhancement } \\
\text { Level (\%) }\end{array}$ \\
\hline \multirow{2}{*}{67} & $3 \mathrm{BS}$ & 128 & \multirow{2}{*}{10.2} \\
\cline { 2 - 3 } & $\begin{array}{c}4 \mathrm{BS} \\
\text { (Enhanced) }\end{array}$ & 115 & \multirow{2}{*}{10.1} \\
\hline \multirow{2}{*}{95} & $3 \mathrm{BS}$ & 175 & \multirow{2}{*}{13.1} \\
\cline { 2 - 3 } & $\begin{array}{c}4 \mathrm{BS} \\
\text { (Enhanced) }\end{array}$ & 152 \\
\hline
\end{tabular}

distance was $115 \mathrm{~m}$ at $67 \%$ deployment, and $152 \mathrm{~m}$ at $95 \%$ attempt of using the model.

The Enhanced hybrid technique improved on the 3 BS case by $10.2 \%$ and $13.1 \%$ at deployment levels of $67 \%$ and $95 \%$ respectively. This delivered an overall $11.6 \%$ improvement of the Enhanced hybrid technique over its 3 BS counterpart.

According to Table 3 and Fig 3, the readings from 3 BS, at $67 \%$ located the MS within a distance of approximately $128 \mathrm{~m}$. While at the confidence level of $95 \%$, the MS was found within $175 \mathrm{~m}$. In the case of 4 BS however, the MS location 
Table 4. Accuracy Result 2: Enhanced Hybrid Technique, TDOA and RSS compared using 4 BS

\begin{tabular}{|c|c|c|c|}
\hline \multirow{2}{*}{ CDF (\%) } & \multicolumn{3}{|c|}{ Location Distance(m) } \\
\cline { 2 - 4 } & HYBRID & RSS & TDOA \\
\hline 67 & 115 & 199 & 189 \\
\hline 95 & 152 & 339 & 300 \\
\hline Enhancement Level (\%) & 48.7 & 44.2 \\
\hline
\end{tabular}

Fig 4 clearly shows the superiority of the Enhanced Hybrid technique over TDOA and RSS. Table 4 reveals a $48.7 \%$ improvement over RSS and $44.2 \%$ improvement over TDOA techniques when compared.

The dataset in Table 2 was separated into four quartiles for a closer analysis. The first quartile slope revealed that at $22.6 \%$ deployment the developed model found the MS within $40 \mathrm{~m}$. At the second quartile, the slope indicated a location distance of $102 \mathrm{~m}$ at a deployment level of $57 \%$. The model traced the MS within a distance of $161 \mathrm{~m}$ at $96 \%$ cumulative probability in the third quartile. The fourth quartile has a slope of $132 \%$ against $284 \mathrm{~m}$ location distance. This deployment level is however beyond the radar of the FCC accuracy requirement for outdoor Network based (Non-Line-Of-Sight) MSP techniques (which focuses mainly on the location distance at $67 \%$ and $95 \%$ cumulative probabilities or deployment levels). However, the results show a steady increase in the location distance as the cumulative probability increased from quartile one to four.

\subsubsection{Latency Result}

In this section, the average time it took to produce the location estimate for each technique is displayed. Latency is the delay experienced when producing a location estimate. In this case, the extra time delay experienced when the BS was increased from 3 to 4 gives the latency. A very high latency value could render a solution inconsequential or totally unworkable notwithstanding a significant increase in accuracy. See Table 5 for comparison.

Table 5. Latency Result

\begin{tabular}{|c|c|c|}
\hline RSS/TDOA METHOD & $\mathbf{3 ~ B S}$ & 4 BS (Enhanced) \\
\hline $\begin{array}{c}\text { Average Elapsed Time } \\
\text { (Seconds) }\end{array}$ & 0.233022 & 0.3013242 \\
\hline Latency (Seconds) & \multicolumn{2}{|c|}{0.068} \\
\hline Percentage Latency & $22.67 \%$ \\
\hline
\end{tabular}

As shown in Table 5, the latency value is 0.068 seconds which makes a $22.67 \%$ increment in time from 3 to 4 BS.

\section{CONCLUSION}

In this study, an enhanced hybrid technique was used to efficiently find the position of a MS in urban and suburban areas. The latency issues that accompanied the increase in number of BS from the conventional 3 to 4 was also investigated. This study showed that the developed technique with 4 BS outperformed the same technique using 3 BS given that no hardware adjustments were made to the currently available handsets. Simulation results showed that $67 \%$ of the readings were within 115 meters of the MS and $95 \%$ of the readings were within 152 meters. Hence, there is up to $11.6 \%$ improvement on the developed hybrid technique over the same technique using $3 \mathrm{BS}$. On the other hand, when compared with the results obtained from the individual techniques (RSS and TDOA with $4 \mathrm{BS}$ ), the developed technique delivered an improvement of $48.7 \%$ over the RSS and $44.2 \%$ over the TDOA.

Furthermore, the latency value (from 3 to 4 BS) was 0.068 seconds. Efforts could still be made to reduce this latency while sustaining the accuracy. The solution presented in this work is valuable to Communication Engineers, football league industry for goal line technology as well as any emergency distress callers. The extra computation loading at the network end can be calmed by using modern powerful computation machines.

\section{FUTURE WORKS}

The following is recommended for future studies on this work:

i. Efforts should be made to reduce latency without compromising accuracy.

ii. Developed techniques should be stretched to locate more than one MS at a time. This would actually improve latency.

iii. More than two techniques could be merged and their performance evaluated.

iv. Other techniques could be employed to perhaps meet the FCC accuracy requirement.

\section{ACKNOWLEDGMENTS}

Our thanks to the Almighty God who has been by our side all through this research. His grace was sufficient to start and finish this work.

\section{REFERENCES}

[1] T. B. Timothy, H. B. Huub, A. M. Ken and H. P. Wyatt, "A Review of Position Tracking Methods," 1st International Conference on Sensing Technology, p. 1, 2012.

[2] A.-R. Sharief, K. Yahya and A.-I. Mohammad, "Mobile Station Positioning using Time Difference of Arrival and Received Signal Strength," International Journal of Mobile Communications, October 2012.

[3] R. S. Campos, "Evolution of Positioning Techniques in Cellular Networks, from $2 \mathrm{G}$ to 4G," Wireless Communications and Mobile Computing, p. 1, 12 January 2017.

[4] J. A. d. Peral-Rosado, R. Raulefs, J. A. L'opez-Salcedo and G. Seco-Granados, "Survey of Cellular Mobile Radio Localization Methods: from 1G to 5G," IEEE Communications Surveys and Tutorials, pp. 9-11, 2017.

[5] A. A. Adeyelu, O. Onah and J. Orban, "An EnhancedRecieved Signal Strength Technique for Estimating Mobile Station Position in Wireless Sensor Networks," Communications on Applied Electronics, vol. 7, no. 33, july 2020 .

[6] A. A. Adeyelu, O. J. Onaji and I. J. Orban, "An Enhanced-Time Difference of Arrival Technique for Estimating Mobile Station Position in Wireless Sensor Networks," International Journal of Computer Applications, October 2020. 
[7] A. D. Gante and M. Siller, "A Survey of Hybrid Schemes for Location Estimation in Wireless Sensor Networks," The 2013 Iberoamerican Conference on Electronics Engineering and Computer Science, 2013.

[8] R. Kaune, "Accuracy Studies for TDOA and TOA Localization," 15th International Conference on Information Fusion, 2012.
[9] R. Kaune, J. Hörst and W. Koch, "Accuracy analysis for TDOA localization in sensor networks," 14th International Conference on Information Fusion, 5-8 July 2011. 